\title{
EFTEM at High Magnification: Principles and Practical Applications
}

\author{
Werner Grogger*, Kannan M. Krishnan**, Ferdinand Hofer* \\ * Research Institute for Electron Microscopy, Graz University of Technology, Steyrergasse 17, A- \\ 8010 Graz, Austria \\ ** NCEM, Materials Sciences Div., Lawrence Berkeley National Lab., Berkeley, CA 94720, USA; \\ present address: Dept. of Materials Sciences, University of Washington, Seattle, WA 98195, USA
}

Energy-filtering TEM (EFTEM) has proven to be an efficient technique for determining elemental distributions on a nanometer length scale. Especially, post-column energy-filters in combination with high resolution TEMs can provide the capability to detect and separate small features down to subnanometer regions [e.g. 1, 2, 3; fig. 1]. Far more than in high-resolution TEM, the attainable spatial resolution is influenced by the experimental setup: The energy and the shape of the ionization edge, as well as experimental parameters like the collection angle, influence the obtainable resolution together with instrumental parameters (aberration coefficients of the TEM objective lens) $[4,5]$. Additionally to these theoretical limitations, however, there are practical aspects, that also limit the resolution in EFTEM: maximum electron dose, exposure time, specimen drift and instrumental instabilities. When considering, that at high magnifications only a small number of atoms (typically $10-1000$ ) contribute to the signal in a pixel of the final image, it is obvious that accurate instrumental alignments and a careful experimental setup are paramount for good quality results. It should also be noted, that a certain amount of elastic contrast is always transferred to energy-filtered images [6], which can lead to artifacts in elemental distribution images.

In this study we present results obtainable with high resolution EFTEM. Fig. 1 shows an elemental map (three window technique) of a $\mathrm{Mn} / \mathrm{PdMn}$ multilayer using the $\mathrm{Mn}-\mathrm{L}_{23}$ ionization edge acquired on a $200 \mathrm{kV}$ FEG-TEM with a post-column energy filter. The Mn layer thicknesses are between 2.6 and $0.47 \mathrm{~nm}$, the thickness of the PdMn spacer layer is constant $(3.7 \mathrm{~nm})$. The integrated profile across the image shows peaks for every single layer. As the layers get thinner, the widths of the peaks remain constant for layer thicknesses below about $1.5 \mathrm{~nm}$. For thinner layers the area under the peak decreases, until it may reach a level, where it cannot be longer distinguished from noise (estimated to be around $0.16 \mathrm{~nm}$ for the acquisition parameters used in fig. 1).

For many applications it is important to characterize thin oxide layers, e.g. in semiconductor devices, which nowadays reach thicknesses of just a few monolayers. The thickness of such layers in semiconductor devices plays an essential role in the functionality of the device. Using the contrast difference between the Si substrate and the thin silicon oxide layer at certain energy losses, the oxide layer can be imaged at $\mathrm{nm}$ resolution (fig. 2). At the low energy-losses used $(30-90 \mathrm{eV})$ the signal to noise ratio is very high, which allows short acquisition times. However, at these low energy-losses delocalization is decreasing the resolution to values of a couple of nm. Our work will be presented how to account for this broadening, and to remove it digitally in order to improve the resolution.

The dimensions in many materials science devices become smaller and smaller, that's why the reliability of compositional profiles is an important issue. EFTEM provides a quick analytical tool 
for such investigations, but in some cases its applicability may be limited due to the limited spatial resolution. In this paper we highlight these limitations as well as the prospects of the technique.

[1] B. Freitag and W. Mader, J. Microsc. 194 (1999) 42

[2] K.T. Moore et al., Phil. Mag. B 82 (2002) 13

[3] M. Varela et al., Phys. Rev. Lett. 86 (2001) 5156

[4] O.L. Krivanek et al., J. Microsc. 180 (1995) 277

[5] R.F. Egerton, J. Electron Microsc. 48 (1999) 711

[6] T. Navidi-Kasmai and H. Kohl, Ultramicroscopy 81 (2000) 223

[7] W.G. acknowledges financial support from the Max Kade foundation for his stay at the NCEM, Berkeley, where part of this work was done. We thank Austriamicrosystems AG, Schloss Premstätten, A-8141 Unterpremstätten, Austria for supplying the semiconductor sample.
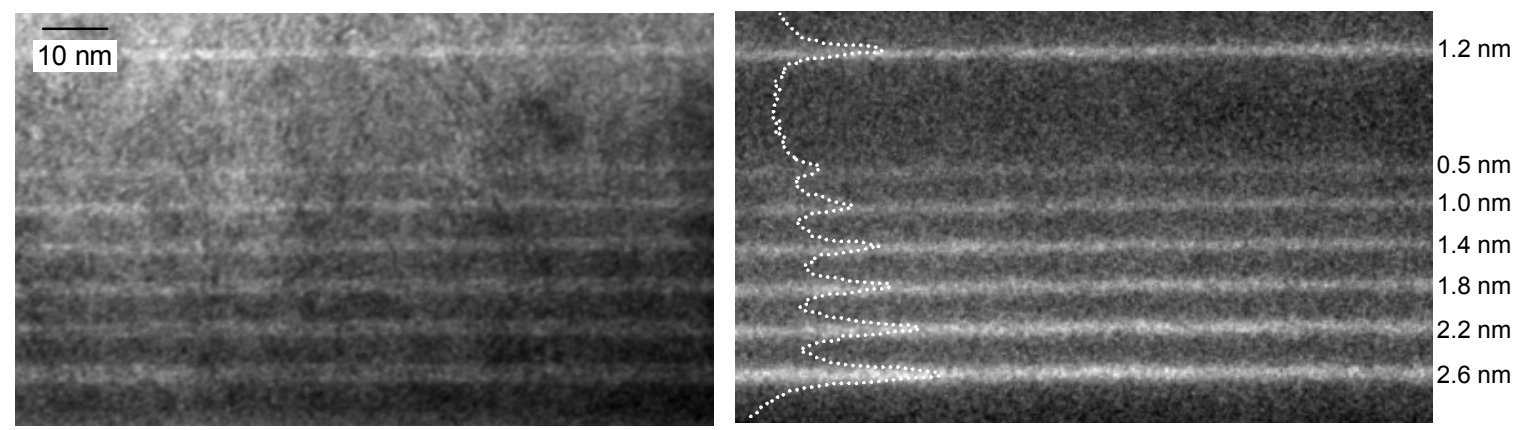

Fig. 1: Bright field image (left) and $\mathrm{Mn}-\mathrm{L}_{23}$ elemental map (right) of a Mn/PdMn multilayer. The thinnest Mn layer is $0.5 \mathrm{~nm}$ thick. The elemental map was calculated from energy-filtered images at 607,627 and $650 \mathrm{eV}$ with a $20 \mathrm{eV}$ slit and an exposure time of $20 \mathrm{~s}$.
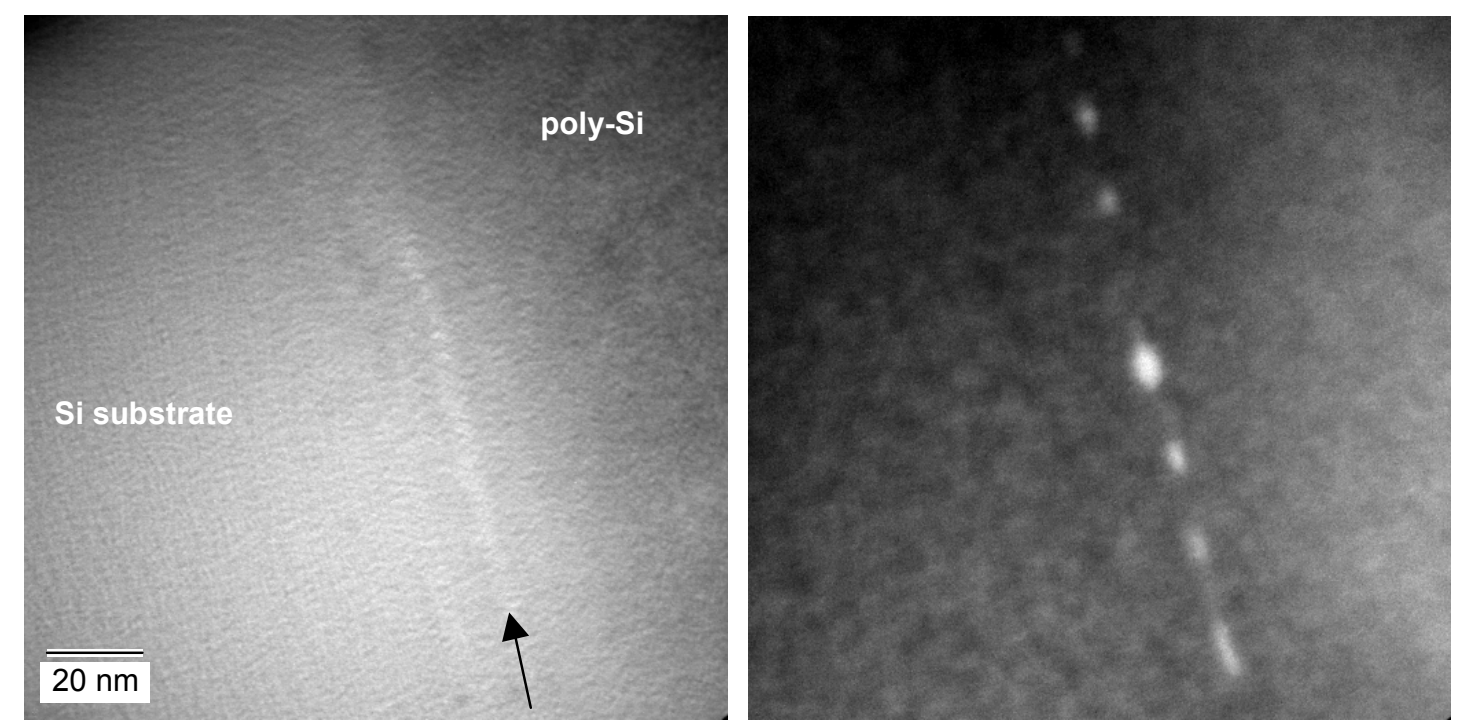

Fig. 2: Bright field image (left) and energy-filtered image at $30 \mathrm{eV}$ (10 eV slit, right) of a thin oxide layer (arrow) on a $\mathrm{Si}$ substrate underneath an originally amorphous silicon layer, which was subsequently recrystallized by annealing at $1000{ }^{\circ} \mathrm{C}$. Using EFTEM the nominally $1 \mathrm{~nm}$ thin oxide layer could be detected and an insular growth confirmed. 